\title{
Mayor beneficio con una meta de presión arterial sistólica menor de $130 \mathrm{mmHg}$
}

\author{
More benefit with systolic pressure target lower than $130 \mathrm{mmHg}$
}

Verdecchia P y col. Lancet. 2009; 374(9689):525-33.

\section{Objetivo}

Comparar el efecto de una meta de presión arterial sistólica (PAS) menor a $140 \mathrm{mmHg}$ (tratamiento estándar) ó a 130 $\mathrm{mmHg}$ (tratamiento intensivo) sobre la incidencia de hipertrofia ventricular izquierda $(\mathrm{HVI})$ en pacientes con hipertensión controlada sin diabetes.

\section{Diseño, lugar y pacientes}

Ensayo clínico aleatorizado realizado en 44 centros de Italia (1111 pacientes).

\section{Medición de resultados principales}

El punto final primario, analizado por intensión de tratar*, fue la prevalencia de $\mathrm{HVI}$ electrocardiográfica a los dos años. El secundario estuvo compuesto por muerte, infarto de miocardio (IM), accidente cerebrovascular (ACV) o isquémico transitorio e insuficiencia cardíaca (IC).

\section{Resultados Principales}

Los desenlaces primarios y secundarios fueron más frecuentes en el grupo de tratamiento estandar, con más fibrilaciones auriculares $(3,8$ vs. $1,8 \%, p=0,044)$ y revascularizaciones $(2,7$ vs. $0,96 \%, p=0,032)$. El número de fármacos no difirió, aunque el grupo intervensión utilizó más intensivamente diuréticos $(36 \%)$ y antagonistas del receptor de angiotensina II $(17 \%)$. La incidencia de efectos secundarios fue baja y $\sin$ diferencias.

Tabla 1: prevalencia a los dos años de hipertrofia ventricular izquierda de acuerdo a la meta del tratamiento.

\begin{tabular}{|c|c|c|c|}
\hline & \multicolumn{2}{|c|}{$\begin{array}{c}\text { Presión arterial sistólica en } \\
\mathrm{mmHG}\end{array}$} & \multirow[t]{2}{*}{ OR (IC 95\%) } \\
\hline & Menor a 130 & Menor a 140 & \\
\hline Prevalencia de HVI a los dos años & $11,4 \%$ & $17 \%$ & $0,63(0,43$ a 0,91$)$ \\
\hline
\end{tabular}

$\mathrm{HVI}$ : hipertrofia ventricular izquierda valorada electrocardiograficamente.

\section{Conclusiones}

Los resultados avalan una meta terapéutica más baja (menor a $130 \mathrm{mmHg}$ ) a la recomendada actualmente en hipertensos no diabéticos.

Palabras claves: hipertensión arterial, metas de presión arterial, control intensivo, hipertrofia ventricular izquierda

Keywords: Hypertension, arterial pressure targets, intensive control, left ventricular hypertrophy.

Fuentes de financiamiento: Boeringer Ingelheim, Sanofi Aventis y Pfizer.

\section{Comentario}

El aumento de la PAS confiere mayor riesgo cardiovascular que el de la diastólica ${ }^{1}$ y a pesar de ello su control suele ser menor $^{2}$. La meta terapéutica óptima a alcanzar genera controversia. Los resultados de diversos ensayos clínicos (UKPDS ${ }^{3}$, $\mathrm{HOT}^{4}, \mathrm{ADVANCE}^{5}$ ) sustentan una meta terapéutica menor en diabéticos (menor a 130/80 $\mathrm{mmHg}$ ) que en no diabéticos (menor a $140 / 90 \mathrm{mmHg}$ ), mientras que un estudio reciente (ACCORD) no mostró ventajas en bajar la PAS a menos de $120 \mathrm{mmHg}$ (contra un descenso menor a $140 \mathrm{mmHg}$ ) en diabéticos. $^{6}$

Sin embargo, el beneficio de un control mayor al usual en los no diabéticos es menos claro y eventualmente perjudicial en muy ancianos ó en pacientes de alto riesgo cardiovascular ${ }^{7}$. Si bien una reciente revisión de siete ensayos clínicos no había encontrado beneficio adicional con metas de presión inferiores a las "estándar";; este ensayo clínico demostró un beneficio adicional respecto de la valoración de un daño de órgano blanco. Es clara la necesidad de combinar dos ó más fármacos para alcanzar el control de la hipertensión sistólica9 ${ }^{9}$ recomendando las guías de hipertensión ${ }^{10}$ combinar dos fármacos cuando la PAS está $20 \mathrm{mmHg}$ por encima de la meta.

\section{Conclusiones del comentador}

Aunque no constituye una evidencia definitiva en términos de morbimortalidad, este trabajo avala utilizar una meta menor a $130 \mathrm{mmHg}$ en hipertensos no diabéticos.

Ver glosario*

José Alfie [ Unidad de Hipertension Arterial, Servicio de Clínica Médica del Hospital Italiano de Buenos Aires jose.alfie@hospitalitaliano.org.ar ]

Alfie J. Mayor beneficio con una meta de presión arterial sistólica menor de 130mmHg. Evid. actual. práct. ambul; 13(2) 48. Abr-Jun 2010. Comentado de Verdecchia $\mathbf{P}$ et al.Usual versus tight control of systolic blood pressure in non-diabetic patients with hypertension (Cardiosis): an open-label randomised trial. Cardio-Sis investigators. Lancet. 2009;374(9689):525-33.PMID: 19683638.

\section{Referencias}

1. Neaton J y col. Serum Cholesterol, Blood Pressure, Cigarette Smoking, and Death From Coronary Heart Disease Overall Findings and Differences by Age for 316099 White Men. Arch Intern Med.1992;152:56-64.

2. Lloyd-Jones D y col. Differential Control of Systolic and Diastolic Blood Pressure. Factors Associated With Lack of Blood Pressure Control in the Community. Hypertension. 2000;36:594-599

3. UK Prospective Diabetes Study Group.Efficacy of atenolol and captopril in reducing risk of macrovascular and microvascular complications in type 2 diabetes: UKPDS 39. BMJ. 1998;317(7160):713-20.

4. HOT Study Group. Effects of intensive blood-pressure lowering and low-dose aspirin in patients with hypertension: principal results of the Hypertension Optimal Treatment (HOT) randomised trial. Lancet. 1998;351(9118):1755-62.

5. Patel A; ADVANCE Collaborative Group. Effects of a fixed combination of perindopril and indapamide on macrovascular and microvascular outcomes in patients with type 2 diabetes mellitus (the ADVANCE trial): a randomised controlled trial. Lancet. 2007; 370(9590):829-40.

6. The ACCORD Study Group. Effects of intensive blood-pressure control in type 2 diabetes mellitus. Nengl J Med. 2010;362:1575-85. M7. Arguedas J y col. Treatment blood pressure targets for hypertension. Cochrane Database of Systematic Reviews 2009, Issue 3. Art. No.: CD004349. DOI: 10.1002/14651858.CD004349.pub2

7. Sleight P,et al.,ONTARGET investigators. Prognostic value of blood pressure in patients with high vascular risk in the Ongoing Telmisartan Alone and in combination with Ramipril Global Endpoint Trial study. J Hypertens. 2009;27(7):1360-9.

8. Arguedas J y col. Treatment blood pressure targets for hypertension. Cochrane Database of Systematic Reviews 2009, Issue 3. Art. No.: CD004349. DOI: 10.1002/14651858.CD004349.pub2

9. Cushman W y col. Achieving blood pressure goals: why aren't we?J Clin Hypertens (Greenwich). 2006;8(12):865-72.

10. Chobanian A y col. and the National High Blood Pressure Education Program Coordinating Committee. Seventh Report of the Joint National Committee on Prevention, Detection, Evaluation, and Treatment of High Blood Pressure. Hypertension, 2003; 42: 1206 - 1252. 